PROCEEDINGS OF THE AMERICAN MATHEMATICAL SOCIETY

Volume 124, Number 8, August 1996

\title{
BASES FOR VECTOR SPACES OVER THE TWO-ELEMENT FIELD AND THE AXIOM OF CHOICE
}

\author{
KYRIAKOS KEREMEDIS
}

(Communicated by Andreas R. Blass)

\begin{abstract}
It is shown that the axiom of choice follows in a weaker form than the Zermelo - Fraenkel set theory from the assertion: every set of generators $G$ of a vector space $V$ over the two element field includes a basis $L$ for $V$. It is also shown that: for every family $\mathcal{A}=\left\{A_{i}: i \in k\right\}$ of non empty sets there exists a family $\mathcal{F}=\left\{F_{i}: i \in k\right\}$ of odd sized sets such that, for every $i \in k$, $F_{i} \subseteq A$ iff in every vector space $B$ over the two-element field every subspace $V \subseteq B$ has a complementary subspace $S$ iff every quotient group of an abelian group each of whose elements has order 2 has a set of representatives.
\end{abstract}

Blass [1] proved, in ZF, that the axiom of choice, $\mathrm{AC}$ (if $A$ is a pairwise disjoint family of non empty sets, then there exists a set $C$ which consists of one and only one element from each element of $A$ ), is deducible from the assertion

$$
\text { Every vector space has a basis. }
$$

It is an open question (see [1], p. 33]) whether (1) can be replaced by the weaker statement

Every vector space over a specific field has a basis.

Halpern [3], working in a weaker theory than ZF (called WZF in [1] and being ZF with the axiom of regularity omitted and the axiom of extensionality weakened so as to admit atoms), deduced $\mathrm{AC}$ from the following assertion:

In every vector space every generating set contains a basis.

Thus, in analogy with (2), one may ask whether (3) can be replaced by the weaker statement

$$
\text { In every vector space over the two-element field every }
$$
generating set contains a basis.

It is also an open question (see [2], Theorem 1) whether AC follows, in WZF, from the statement:

In every vector space $B$ over the two-element field, every subspace $V \subseteq B$ has a complementary subspace $S$.

The research in this paper is motivated by the above mentioned questions. We show in Theorem 1 that, in WZF, AC follows from (4), and we show in Theorem 2 that, also in WZF, the odd axiom of choice, OAC, i.e., the statement:

Received by the editors June 21, 1993 and, in revised form, February 16, 1995.

1991 Mathematics Subject Classification. Primary 03E25.

(C)1996 American Mathematical Society 
For every family $\mathcal{A}=\left\{A_{i}: i \in k\right\}$ of non empty sets there exists a family $\mathcal{F}=\left\{F_{i}: i \in k\right\}$ of odd sized sets such that for every $i \in k$ $F_{i} \subseteq A$

is equivalent to (5). (OAC is actually equivalent to the statement MC 4(2) from [5], p. 83, and it is due to M. N. Bleicher [2].)

In Theorem 3, we show, in WZF, that AC is equivalent to the combination of the statements $\mathrm{OAC}$ and $\mathrm{EAC}$, where $\mathrm{EAC}$ is the statement given by:

For every family $\mathcal{A}=\left\{A_{i}: i \in k\right\}$ of non empty sets with $\left|A_{i}\right|>1$ there exists a family $\mathcal{F}=\left\{F_{i}: i \in k\right\}$ of non empty even sized sets such that, for every $i \in k, F_{i} \subseteq A_{i}$.

Finally, in Theorem 4 we show, in $\mathrm{ZF}$, that $\mathrm{AC}$ is equivalent to the statement:

Every quotient group of an abelian group each of whose elements has order 2 has a set of representatives.

Since AC is expressed in terms of sets, it is only natural to work with vector spaces whose elements as well as scalars are sets, and, as expected, addition, $\oplus$, and scalar multiplication, $\bullet$, are symmetric difference and set-theoretic intersection, respectively. Since the set of scalars has to be a field under the above mentioned operations, we see that it must be the two-element field.

We are obliged to Professor Andreas Blass for several very useful and intriguing remarks concerning Theorems 1 and 3.

Theorem 1. In WZF, AC is deducible from the assertion that in every vector space $B$ over the two-element field, every generating set contains a basis.

Proof. Fix $\mathcal{A}=\left\{A_{i}: i \in k\right\}$, a family of non empty sets. Without loss of generality we may assume that $A_{i} \cap A_{j}=\emptyset$ and $A_{i}$ is infinite for all $i \neq j, i, j \in k$. For every $i \in k$, define

$$
\mathbf{A}_{i}=\left\{a \subseteq A_{i}: a \text { or } A_{i} \backslash a \text { is finite }\right\} .
$$

It is straightforward to see that $\left(\mathbf{A}_{i}, \oplus\right)$, where $\oplus$ denotes the operation of symmetric difference, is an abelian group in which all elements satisfy

$$
x \oplus x=0 .
$$

As is well known, a vector space over the two-element field is the same thing as an abelian group each of whose elements satisfies the equation

$$
x+x=0 .
$$

Thus, $\left(\mathbf{A}_{i}, \oplus\right)$ is a vector space over the two-element field. From now on, we shall treat vector spaces over the two-element field as abelian groups satisfying (7).

Let $\bar{A}$ denote the direct sum of the family $\left\{\mathbf{A}_{i}: i \in k\right\}$. Then $(\bar{A}, \oplus)$, where $\oplus: \bar{A} \times \bar{A} \rightarrow \bar{A}$ is given by

$$
(\bar{a} \oplus \bar{b})(i)=\bar{a}(i) \oplus \bar{b}(i),
$$

is an abelian group satisfying (7).

Let $G=\bigcup\left\{D_{j a}: j \in k, a \in \mathbf{A}_{j}\right\}$, where

$$
D_{j a}=\left\{\bar{a} \in \bar{A}: \bar{a}(i)=\emptyset \text { if } i \neq j \text { and } \bar{a}(i) \in\left\{\{a\}, A_{i} \backslash\{a\}\right\} \text { otherwise }\right\} .
$$

It is straightforward to see that $G$ is a set of generators for $\bar{A}$. By the assertion of the theorem, there exists a basis $L \subseteq G$ for $\bar{A}$. 
For every $i \in k$, let $\bar{a}_{i} \in \bar{A}$ be the element given by:

$$
\bar{a}_{i}(j)=A_{i} \text { if } i=j \text { and } \bar{a}_{i}(j)=\emptyset \text { otherwise. }
$$

Claim 1. For every $i \in k$ there is a unique $y_{i} \in A_{i}$ such that $\left\{y_{i}\right\}^{*} \in L$ and $\left(\left\{y_{i}\right\}^{c}\right)^{*} \in L$, where $\left\{y_{i}\right\}^{*}$ and $\left(\left\{y_{i}\right\}^{c}\right)^{*}$ denote the elements of $\bar{A}$ which equal $\left\{y_{i}\right\}$ and $\left\{y_{i}\right\}^{c}$ (= the complement of $\left\{y_{i}\right\}$ with respect to $A_{i}$ ) at $i$ and $\emptyset$ elsewhere, respectively.

Proof of Claim 1. Fix $i \in k$. Let

$$
\bar{a}_{i}=\bar{\ell}_{0} \oplus \bar{\ell}_{1} \oplus \ldots \oplus \bar{\ell}_{m}
$$

be the unique expression of $\bar{a}_{i}$ as a finite linear combination of elements from $L$. Let

$$
\mathcal{J}=\left\{v \leq m:\left|\bar{\ell}_{v}(i)\right| \text { is infinite }\right\} .
$$

Note that $|\mathcal{J}|$ cannot be an even number because the symmetric difference of $\left\{\bar{\ell}_{v}(i)\right.$ : $v \in \mathcal{J}\}$ will be a finite set, and consequently $A_{i}=\bar{a}_{i}(i)$ will be finite since the remaining vectors will only contribute a finite set. If $|\mathcal{J}|$ is odd, then the symmetric difference of

$$
\left\{\bar{\ell}_{v}(i): v \in \mathcal{J}\right\}
$$

will have a finite complement, say $\mathbf{c}$. Thus, for every element $c \in \mathbf{c}$ there must be a $u \leq m$ and a $w \in \mathcal{J}$ with

$$
\bar{\ell}_{u}(i)=\{c\} \text { and } \bar{\ell}_{w}(i)=A_{i} \backslash\{c\} .
$$

(If $c \in \bar{\ell}_{v}(i)$ for all $v \in \mathcal{J}$, then $c \in \bigoplus_{v \in \mathcal{J}} \bar{\ell}_{v}(i)$ because $|\mathcal{J}|$ is odd, but this contradicts $c \in \mathbf{c}$.) Thus, $\bar{a}_{i}=\bar{\ell}_{u} \oplus \bar{\ell}_{w}$. This means that in (8) $m$ must be equal to 1 , and the conclusion of the claim now follows from the fact that $L \subseteq G$ and $L$ is a basis (needed for the uniqueness part). On the basis of the claim we define

$$
C=\left\{y_{i}: i \in k\right\} .
$$

Then $C$ is a choice set for $\mathcal{A}$, finishing the proof of Theorem 1 .

Theorem 2. OAC iff (5).

Proof. $(\leftarrow)$ Fix $\mathcal{A}=\left\{A_{i}: i \in k\right\}$, a family of non empty sets. Let $\bar{A}$ be as in Theorem 1 and

$$
V=\{a \in \bar{A}:|a(i)|=\text { even natural number for every } i \in k\} .
$$

It is straightforward to see that $V$ is a subspace of $\bar{A}$. Let $S$ be its complementary subspace.

Claim 2. For every $i \in k$ there is a unique element $s_{i} \in S$ such that

$$
\begin{aligned}
& \left|s_{i}(i)\right|=\text { odd natural number and } \\
& \left|s_{i}(j)\right|=\text { even natural number for all } j \in k, j \neq i .
\end{aligned}
$$


Proof of Claim 2. Fix $y \in \bar{A}$ such that:

$$
|y(i)|=\text { odd natural number and } y(j)=\emptyset \text { for all } j \in k, j \neq i .
$$

Since $S$ and $V$ are complementary subspaces, there exist elements $s \in S$ and $v \in V$ such that

$$
y=s \oplus v .
$$

Since all coordinates of $v$ are even sized sets, it can be readily verified that $s$ satisfies (10). We note that there can be no two distinct such $s$ 's, for their non zero sum would be a member of $V$. This finishes the proof of the claim.

Put

$$
\mathcal{F}=\left\{F_{i}=s_{i}(i): i \in k\right\}
$$

Clearly $\mathcal{F}$ is as required.

$(\rightarrow)$ This is Theorem 6.37 from [5], p. 124 .

The next theorem tells us how much is missing from the OAC in order to recover AC.

Theorem 3. In $W Z F, A C$ is deducible from $O A C+E A C$.

Proof. Fix $\mathcal{A}=\left\{A_{i}: i \in k\right\}$, a disjoint family of non empty sets. By OAC we may assume that $\mathcal{A}$ is a family of odd sized sets. Let

$$
E=\{x \in \mathcal{P}(\cup \mathcal{A}) \backslash\{\emptyset\}: x \text { is even sized }\}
$$

and

$$
O=\{x \in \mathcal{P}(\cup \mathcal{A}):|x|>1, x \text { is odd sized }\} .
$$

Apply $\mathrm{OAC}$ and $\mathrm{EAC}$ to the sets $E$ and $O$, respectively, to get functions $G: E \rightarrow O$ and $Q: O \rightarrow E$ such that:

$$
\forall x \in E G(x) \subset x \text { and } \forall x \in O Q(x) \subset x .
$$

For every $i \in k$ we iterate $G$ and $Q$ alternately until we reach a singleton, say $c_{i}$. Then $c=\left\{c_{i}: i \in k\right\}$ is a choice set for $\mathcal{A}$ as required.

Next, we find another algebraic characterization of OAC. Let $(G,+)$ be an abelian group. We recall that a subgroup $H$ of $G$ partitions $G$ into pairwise disjoint equivalence classes, the so-called cosets. Denoting the equivalence class of an element $g \in G$ by $[g]$, we have:

$$
[g]=\{g+h: h \in H\} .
$$

$g$ is called a representative of the coset $[g]$. We denote the set of all cosets by $G / H$. We also recall that $G / H$ is an abelian group if addition $\oplus$ is given by:

$$
[g] \oplus[q]=[g+q] \text { for every element } g, q \in G .
$$

$(G / H, \oplus)$ is called the quotient group of $G$ modulo $H$.

Theorem 4. OAC iff every quotient group of an abelian group each of whose elements has order 2 has a set of representatives. 
Proof. $(\leftarrow)$ Fix $A=\left\{A_{i}: i \in k\right\}$, a disjoint family of non empty sets. Let $G=\bar{A}$ where $\bar{A}$ is as in Theorem 1 , and let $H$ be the $V$ of (9). For every $i \in k$ put

$$
\begin{aligned}
& G_{i}=\left\{a \in G:\left|a \cap A_{i}\right| \text { is an odd sized set and }\left|a(j) \cap A_{j}\right|\right. \\
& \text { is an even sized set for all } j \in k, j \neq i\} .
\end{aligned}
$$

Then $G_{i} \in G / H$. (A representative for $G_{i}$ will be, for example, any $a \in G$ such that $a(i)$ is a single element subset of $A_{i}$ and $a(j)=\emptyset$ for all $j \in k, j \neq i$.) By the hypothesis, there exists a set of representatives $\mathcal{G} \subseteq G$ such that $G$ is the disjoint union of their corresponding cosets. Let $g_{i} \in \mathcal{G}$ denote the representative of the $\operatorname{coset} G_{i}$ for all $i \in k$. We put

$$
F_{i}=g_{i}(i) \cap A_{i} .
$$

Clearly $F_{i}$ is an odd sized set for every $i \in k$, as required.

$(\rightarrow)$ To see this, fix $G$ to be an abelian group each of whose elements has order 2 and let $H$ be a subgroup of $G$. Let $C=\left\{C_{j}: j \in J\right\}=G / H-\{[0]\}$ and $F=\left\{F_{j}: j \in J\right\}$ be the set which is guaranteed by OAC. Let $g_{j}$ denote the sum of the elements of $F_{j}$. Since $F_{j}$ is odd sized, we see that $g_{j} \in C_{j}$, and consequently $\mathcal{G}=\left\{g_{j}: j \in J\right\}$ is the required set of representatives, finishing the proof of $(\rightarrow)$ and of the theorem.

\section{REFERENCES}

1. A. Blass, Existence of bases implies the axiom of choice, in Axiomatic Set Theory, Contemporary Mathematics, 31 (1984) 31 - 33. MR 86a:04001

2. M. Bleicher, Some theorems on vector spaces and the axiom of choice, Fund. Math. 54 (1964), 95 - 107. MR 29:2190

3. J. D. Halpern, Bases for vector spaces and the axiom of choice, Proc. Amer. Math. Soc., 17 (1966) 670 - 673. MR 33:2550

4. T. Jech, The axiom of choice, North-Holland, 1973. MR 53:139

5. H. Rubin and J.E. Rubin, Equivalents of the Axiom of Choice, II, North-Holland, 1985. MR 87c:04004

University of the Aegean, Department of Mathematics, Karlovasi 83200, Samos, Greece

E-mail address: kker@kerkis.aegean.gr 